\title{
Popular Diets: Ketogenic Diet ${ }^{1}$
}

\section{Kelsey Gemmill, Daniela Rivero-Mendoza, and Wendy Dahl²}

The ketogenic diet is a high-fat, very low carbohydrate diet. The first documented use of the ketogenic diet was in 1921 to treat epilepsy in children (Kim 2017). In the past few years, the ketogenic diet has gained a resurgence as a potential means for weight loss (Harvey, Holcomb, and Kolwicz 2019). The ketogenic diet has become popular due to celebrity endorsement and social media influences, but is it safe and effective?

\section{What is a ketogenic diet?}

The ketogenic diet promotes a high fat intake (up to $90 \%$ of total energy), moderate protein ( $1 \mathrm{~g} / \mathrm{kg}$ body weight/day), and very low carbohydrate intake-less than $50 \mathrm{~g}$ per day (Veyrat-Durebex et al. 2018). The classical ketogenic diet is 4:1 diet, which provides 4 grams of fat for every 1 gram of protein and carbohydrates combined (Fedorovich, Voronina, and Waseem 2018). In the management of epilepsy, the ratio may vary and provide less fat (e.g., 3.75:1). A ketogenic diet may be isocaloric-meaning that it provides adequate calories to maintain body weight-or low or very low calorie, which if adhered to would result in weight loss (Trimboli et al. 2020). A diet high in fat and very low in carbohydrates will force the body into ketosis, a metabolic state in which fat provides most of the fuel (Veyrat-Durebex et al. 2018).

\section{What is ketosis?}

Under normal circumstances, the human body preferably uses glucose for energy (Fedorovich, Voronina, and
Waseem 2018). Once food containing carbohydrates is consumed and digested, the carbohydrate is absorbed as sugar-mostly glucose. The Recommended Dietary Allowance (RDA) for carbohydrate is 130 grams per day for adults, thought to be adequate for glucose provision to the brain and nervous system (Institute of Medicine, Food and Nutrition Board 2005). However, under certain circumstances such as starvation or very low carbohydrate intake, there is not enough glucose to give the body the energy it needs (Fedorovich, Voronina, and Waseem 2018). Instead, the human body has the ability to adapt to these changes and make usable energy in the form of ketone bodies from fat (Fedorovich, Voronina, and Waseem 2018). When the body is relying on ketone bodies for energy, it is in a state of "ketosis" (Fedorovich, Voronina, and Waseem 2018). During fasting or when consuming a ketogenic diet, ketones can be used for energy (Fedorovich, Voronina, and Waseem 2018).

There is a difference between ketosis, which is the result of the ketogenic diet or starvation, and diabetic ketoacidosis. Healthy individuals in ketosis can use ketones for energy, and ketone levels do not reach an unhealthy level in the blood (Fedorovich, Voronina, and Waseem 2018). Diabetic ketoacidosis occurs in people with diabetes when blood glucose becomes very high but cannot be taken up or utilized due to a lack of insulin (Fedorovich, Voronina, and Waseem 2018). Fat is broken down for energy, and ketones build up in the blood to very unsafe levels (Kanikarla-Marie and Jain 2016). Diabetic ketoacidosis is a serious medical condition that can lead to coma and death (Kanikarla-Marie and Jain 2016) and should not be confused with ketosis.

1. This document is FSHN20-44, one of a series of the Food Science and Human Nutrition Department, UF/IFAS Extension. Original publication date September 2020. Visit the EDIS website at https://edis.ifas.ufl.edu for the currently supported version of this publication.

2. Kelsey Gemmill, former MS-DI student; Daniela Rivero-Mendoza, Extension and research coordinator; and Wendy Dahl, associate professor, Food Science and Human Nutrition Department, UF/IFAS Extension; Gainesville, FL 32611.

The Institute of Food and Agricultural Sciences (IFAS) is an Equal Opportunity Institution authorized to provide research, educational information and other services

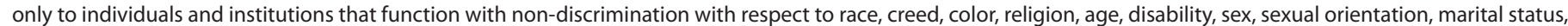

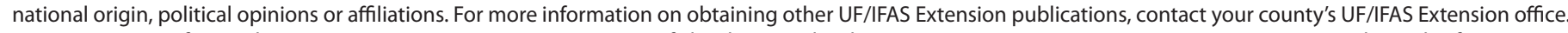
U.S. Department of Agriculture, UF/IFAS Extension Service, University of Florida, IFAS, Florida A \& M University Cooperative Extension Program, and Boards of County Commissioners Cooperating. Nick T. Place, dean for UF/IFAS Extension. 


\section{What's on the menu for a ketogenic diet?}

Foods that contain little to no carbohydrate, such as meats, poultry, fish, eggs, cheese, green leafy vegetables, nuts, oil, butter, mayonnaise, and cream (USDA 2020) are commonly consumed on a ketogenic diet. Even though unsaturated fats, which are considered healthy, are allowed in the ketogenic diet, saturated fats, associated with cardiovascular risk, are also encouraged in this dietary pattern. Nonnutritive sweeteners may be used. Foods that are to be avoided due to their higher content of carbohydrate include bread, cereals, fruits, starchy vegetables, baked goods, and legumes (e.g., beans, lentils, and peas) (USDA 2020). Commonly, vegetables are restricted to leafy greens, broccoli, cauliflower, peppers, onions, cucumber, celery, and other vegetables low in carbohydrate. Fruits are much more restricted because of their higher content of carbohydrate, compared to nonstarchy vegetables. It is important to remember that many of the foods restricted in the ketogenic diet are the health-enhancing foods, recommended by the Dietary Guidelines for Americans, such as whole grains, fruits, vegetables, and low-fat dairy (USDA 2015).

\section{Is the ketogenic diet the same as the Atkins diet?}

Both the ketogenic diet and the Atkins diet restrict carbohydrate intake. However, the ketogenic diet also restricts protein intake to just enough to meet needs, whereas the Atkins diet does not restrict protein.

\section{What are the potential health benefits of a ketogenic diet?}

The ketogenic diet is beneficial for the treatment of some intractable epilepsy syndromes (seizure disorders) (D’Andrea Meira et al. 2019). Its potential beneficial effects in adults with obesity, diabetes, and neurological diseases are currently being studied. In a study of overweight adults with type 2 diabetes, the ketogenic diet, over 32 weeks, improved glycemic control and resulted in higher weight loss compared to a low-fat dietary program for diabetes (Saslow et al. 2017). Most of the studies of the ketogenic diet and outcomes related to neurological disease have been conducted in laboratory animals (McDonald and Cervenka 2018), and thus the results cannot be applied to humans.

How might the ketogenic diet facilitate weight loss? One theory is related to the satiating effect of fat, suggesting that a high-fat diet reduces appetite and thereby decreases overall food intake. However, results of studies analyzing the satiating effects of fat have recently suggested otherwise (Warrilow et al. 2019). Another proposed mechanism is that ketogenic diets maintain usual resting energy expenditure-the amount of energy spent on physiological functions such as breathing, brain function, and a beating heart (Gershuni, Yan, and Medici 2018). Frequently, losing weight results in a decrease in resting energy expenditure, which, in turn, makes it easier to regain weight. When following a ketogenic diet that provides fewer calories than are expended, the body will burn more fat for energy than it will accumulate, which may lead to higher loss of fat mass while maintaining lean mass (Gershuni, Yan, and Medici 2018). More research is needed to confirm the metabolic effects of a ketogenic diet.

Because fats are high in energy ( 9 versus 4 calories per gram for protein or carbohydrate), care is needed to not overconsume calories, which would lead to weight gain. To keep calorie intake in check, high-fat, energy-dense foods must be limited to small portions. Consultation with a registered dietitan nutritionist is advised to properly plan ketogenic diets for weight maintenance or weight loss.

\section{What are the risks of a ketogenic diet?}

The typical American diet consumed by adults is much higher in carbohydrate (46\%-48\% of total energy) and lower in fat (34\%-35\% of total energy) than the ketogenic diet (National Center for Health Statistics, Centers for Disease Control and Prevention 2017). Due to restrictions on whole grain, fruit, and vegetable intake, the ketogenic diet is typically very low in dietary fiber and certain vitamins and minerals found in fortified grains and fresh produce (Gordon 2019). Following the ketogenic diet compared to a higher-carbohydrate diet will lead to changes in nutrient intakes and may lead to nutrient deficiencies in the long term (McSwiney and Doyle 2019). Constipation is frequent complaint of the ketogenic diet because this diet restricts high-fiber foods such as whole grains, fruits and some vegetables.

The ketogenic diet may have negative health and wellness effects. In the short term, some individuals following a ketogenic diet have reported flu-like symptoms, including headache, fatigue, nausea, dizziness, gastrointestinal disorders, and decreased energy, among others (Bostock et al. 2020). Lethargy, dehydration, acidosis, mood changes, and increases in infections, vomiting, and constipation have been reported during the first days of usage of ketogenic diet in some children with epilepsy (Vining et al. 1998).

In the long term, a ketogenic diet may contribute to cardiovascular disease due to its high content of saturated fats. In the Dietary Guidelines for Americans, it is recommended 
to consume less than 10 percent of calories from saturated fats, because replacing saturated fats with unsaturated fats may reduce the risk of cardiovascular disease (USDA 2015). It is very unlikely one could achieve this recommendation by following a ketogenic diet. Furthermore, due to its high fat content, ketogenic diet is not recommended for people with certain liver conditions, pancreatic disease, thyroid problems, or gallbladder disease, or for people who have had their gallbladders removed (Gordon 2019). Reports have described kidney stones developing in children on a ketogenic diet (Choi et al. 2010).

\section{Summary}

The ketogenic diet is a very high fat, low-carbohydrate diet. Following a ketogenic diet induces a state of ketosis, where fat is burned as the primary energy source. While the ketogenic diet may be beneficial as part of the medical management of individuals affected by epilepsy syndromes, its efficacy for long-term weight loss and treating neurological diseases has not been proven, and its risks, especially in long-term, are not clear. In the short term, a calorierestricted, ketogenic diet may lead to weight loss similar to other restrictive diets. Overall, the risks of the ketogenic diet may outweigh the potential health benefits. Talk to your health care provider before adopting a ketogenic diet.

\section{References}

Bostock, E. C. S., K. C. Kirkby, B. V. Taylor, and J. A. Hawrelak. 2020. "Consumer Reports of 'Keto Flu' Associated with the Ketogenic Diet." Frontiers in Nutrition 7(20). https://doi.org/10.3389/fnut.2020.00020

Choi, J. N., J. E. Song, J. I. Shin, H. D. Kim, M. J. Kim, and J. S. Lee. 2010. "Renal Stone Associated with the Ketogenic Diet in a 5-Year-Old Girl with Intractable Epilepsy." Yonsei Medical Journal 51 (3): 457-9.

D’Andrea Meira, I., T. T. Romão, H. J. Pires do Prado, L. T. Krüger, M. E. P. Pires, and P. O. da Conceição. 2019. "Ketogenic Diet and Epilepsy: What We Know So Far." Frontiers in Neuroscience 13:5.

Fedorovich, S. V., P. P. Voronina, and T. V. Waseem. 2018. "Ketogenic Diet versus Ketoacidosis: What Determines the Influence of Ketone Bodies on Neurons?" Neural Regeneration Research 13 (12): 2060-3.

Gershuni, V. M., S. L. Yan, and V. Medici. 2018. "Nutritional Ketosis for Weight Management and Reversal of Metabolic Syndrome." Current Nutrition Reports 7 (3): 97-106.
Gordon, B. “What Is the Ketogenic Diet?” 2019. https:// www.eatright.org/health/weight-loss/fad-diets/what-is-theketogenic-diet [Accessed 24th July 2020].

Harvey, K. L., L. E. Holcomb, and S. C. Kolwicz. 2019. "Ketogenic Diets and Exercise Performance." Nutrients 11 (10): 2296.

Institute of Medicine, Food and Nutrition Board. 2005. Dietary Reference Intakes for Energy, Carbohydrate, Fiber, Fat, Fatty Acids, Cholesterol, Protein, and Amino Acids. Washington, D.C.: The National Academies Press.

Kanikarla-Marie, P., and S. K. Jain. 2016. "Hyperketonemia and Ketosis Increase the Risk of Complications in Type 1 Diabetes." Free Radical Biology and Medicine 95:268-77.

Kim, J-M. 2017. "Ketogenic Diet: Old Treatment, New Beginning." Clinical Neurophysiology Practice 2:161-2.

McDonald, T. J. W., and M. C. Cervenka. 2018. "Ketogenic Diets for Adult Neurological Disorders." Neurotherapeutics 15 (4): 1018-31.

McSwiney, F. T., and L. Doyle. 2019. "Low-Carbohydrate Ketogenic Diets in Male Endurance Athletes Demonstrate Different Micronutrient Contents and Changes in Corpuscular Haemoglobin over 12 Weeks." Sports (Basel) 7 (9). https://www.mdpi.com/2075-4663/7/9/201

National Center for Health Statistics, Centers for Disease Control and Prevention. 2019. "Dietary Intake for Adults Aged 20 and Over." https://www.cdc.gov/nchs/fastats/diet. htm [Accessed 24th July 2020].

Saslow, L. R., A. E. Mason, S. Kim, V. Goldman, R. PloutzSnyder, H. Bayandorian et al. 2017. "An Online Intervention Comparing a Very Low-Carbohydrate Ketogenic Diet and Lifestyle Recommendations versus a Plate Method Diet in Overweight Individuals with Type 2 Diabetes: A Randomized Controlled Trial." Journal of Medical Internet Research 19 (2): e36.

Trimboli, P., M. Castellana, D. Bellido, and F. F. Casanueva. 2020. "Confusion in the Nomenclature of Ketogenic Diets Blurs Evidence." Reviews in Endocrine and Metabolic Disorders. 21 (1): 1-3.

United States Department of Agriculture (USDA). 2015. Dietary Guidelines for Americans 2015-2020. https:// health.gov/our-work/food-nutrition/2015-2020-dietaryguidelines/guidelines/ [Accessed 24th July 2020]. 
United States Department of Agriculture. 2020. FoodData

Central. https://fdc.nal.usda.gov/index.html [Accessed 24th

July 2020].

Veyrat-Durebex, C., P. Reynier, V. Procaccio, R.

Hergesheimer, P. Corcia, C. R. Andres et al. 2018. "How

Can a Ketogenic Diet Improve Motor Function?" Frontiers

in Molecular Neuroscience 11 (15). https://doi.org/10.3389/

fnmol.2018.00015

Vining, E. P. G., J. M. Freeman, K. Ballaban-Gil, C. S.

Camfield, P. R. Camfield, G. L. Holmes et al. 1998. "A Multicenter Study of the Efficacy of the Ketogenic Diet." Archives of Neurology 55 (11): 1433-7.

Warrilow, A., D. Mellor, A. McKune, and K. Pumpa. 2019.

"Dietary Fat, Fibre, Satiation, and Satiety-a Systematic

Review of Acute Studies." European Journal of Clinical

Nutrition 73 (3): 333-44. 